\title{
MEG Modeling and Measurements of Optical Antenna Sensors as Microrectangular Apertures
}

\author{
Alessandro Massaro and Roberto Cingolani
}

\begin{abstract}
In this paper, we present a novel model for the design of micrometric optical antennas. The accuracy of the model allows to evaluate the near field generated by a micrometric aperture in parallel-plate waveguides. The model starts from the equivalent source distribution of magnetic current excitation of a rectangular aperture and it proceeds by employing the multipole expansion of the Green's function applied to the aperture, including the analysis of the electromagnetic field in proximity of metallic discontinuities. The presented method introduces the novel principle of simultaneous transverse resonance diffraction which determines the order of singularity for different edges at optical frequencies. The theory is validated by numerical simulations and experimental results yielding information about the accuracy of the radiated field solution. The approach is suitable for inclusion in standard electromagnetic simulators.
\end{abstract}

Index Terms-Diffraction, modeling, near field, optical antennas, optical circuits, waveguides.

\section{INTRODUCTION}

$\mathbf{O}$ PTICAL antennas are an emerging concept in physical optics. Similar to radiowave and microwave antennas, their purpose is to convert the energy of free propagating radiation to localized energy, and vice versa. In optical science and engineering, light is commonly controlled by redirecting the wavefronts of propagating radiation by means of lenses, mirrors, and diffractive elements. This type of manipulation relies on the wave nature of electromagnetic fields and is therefore not amenable to controlling fields on the subwavelength scale. In contrast, radiowave and microwave technology predominantly makes use of antennas to manipulate electromagnetic fields, controlling them on the subwavelength scale and interfacing efficiently between propagating radiation and localized fields. While antennas are a key enabling technology for devices like cellular phones and televisions using electromagnetic radiation in the radiowave or microwave regime, their optical analog is basically nonexistent in today's technology. However, recent research in nano-optics [1]-[3] and plasmonics [4] has generated considerable interest in the optical antenna concept [5], [6],

Manuscript received November 02, 2010; revised February 22, 2011 and March 21, 2011; accepted April 08, 2011. Date of publication April 15, 2011; date of current version June 03, 2011.

A. Massaro is with the Center of Biomolecular Nanotechnologies of Arnesano, Italian Institute of Technology, Leece 73100, Italy (e.mail: alessandro. massaro@iit.it).

R. Cingolani is with the Italian Institute of Technology, Genova 16163, Italy (e-mail: rob.cingo@unile.it).

Color versions of one or more of the figures in this paper are available online at http://ieeexplore.ieee.org.

Digital Object Identifier 10.1109/JLT.2011.2143388 and several studies are currently focused on how to translate established radiowave and microwave antenna theories into the optical frequency regime [7]-[9]. The micro/nano scale of antennas requires an accurate modeling especially for near-field applications. The traditional numerical approaches such as finite element method (FEM), and finite-difference time-domain technique require high computational cost for the evaluation of the radiated field in proximity of a dielectric or metallic discontinuity. For this purpose, we use the new approach of multipole expansion of the Green's function (MEG) based on the novel principle of simultaneous transverse resonance diffraction (STRD) [10]. The new approach was previously applied for the near-field evaluation of 3-D dielectric corner by showing a good convergent solution with a low computational cost with a reduction of the computational time of 15/12 times if compared with the traditional numerical methods [10]. For the first time we apply the MGE method to a particular class of antennas obtained as slot apertures in parallel-plate waveguides [11], [12]. The use parallel-plate waveguides in optics presents various advantages such as easy fabrication process (deposition of metallic layers on both side of a standard glass for chemical sample), the use of simplified experimental setups, and the applicability in a large number of applications for sensing wireless detection systems especially for robotics and biomedical sensing. Moreover, the use of micrometric apertures on the waveguides allows to use the device in small spaces useful for implanted systems and aerospace wireless applications. However, by considering high frequencies in the optical frequency band, the modeling of the radiation field coming from these apertures becomes difficult to improve. For this purpose, we propose in this paper the MEG approach which evaluates the radiation field, generated by a micromectric slot aperture in parallel-plate waveguides, with a good accuracy and low computational. We list the arguments analyzed in this paper as follows. In particular we:

1) provide parallel-plate waveguide theory at optical frequencies, including radiation aspects of a micrometric aperture;

2) apply the STRD method for the evaluation of the near field generated by optical rectangular apertures in parallel-plate waveguides;

3) analyze the advantages of the layout of Fig. 1, by comparing a near-field permittivity detection system constituted by two metallic $10^{\circ}$ corners with a rectangular aperture detection system;

4) calculate the far field generated of a rectangular aperture by comparing the MEG results with 3-D FEM ones;

5) compare experimental results with 3-D FEM and MEG ones in order to prove the convergence of the solutions. 


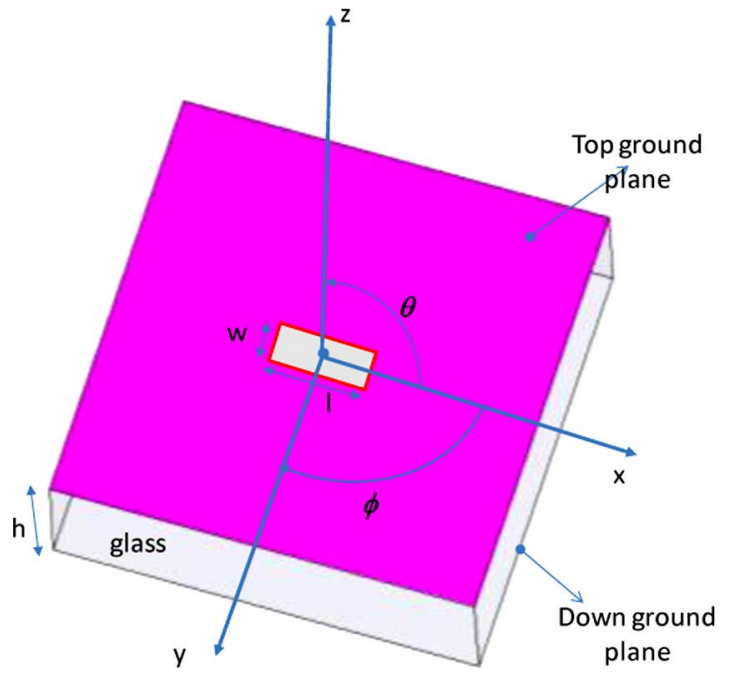

Fig. 1. Optical antenna as micrometric aperture in parallel-plate waveguide. The aperture is in the top ground plane.
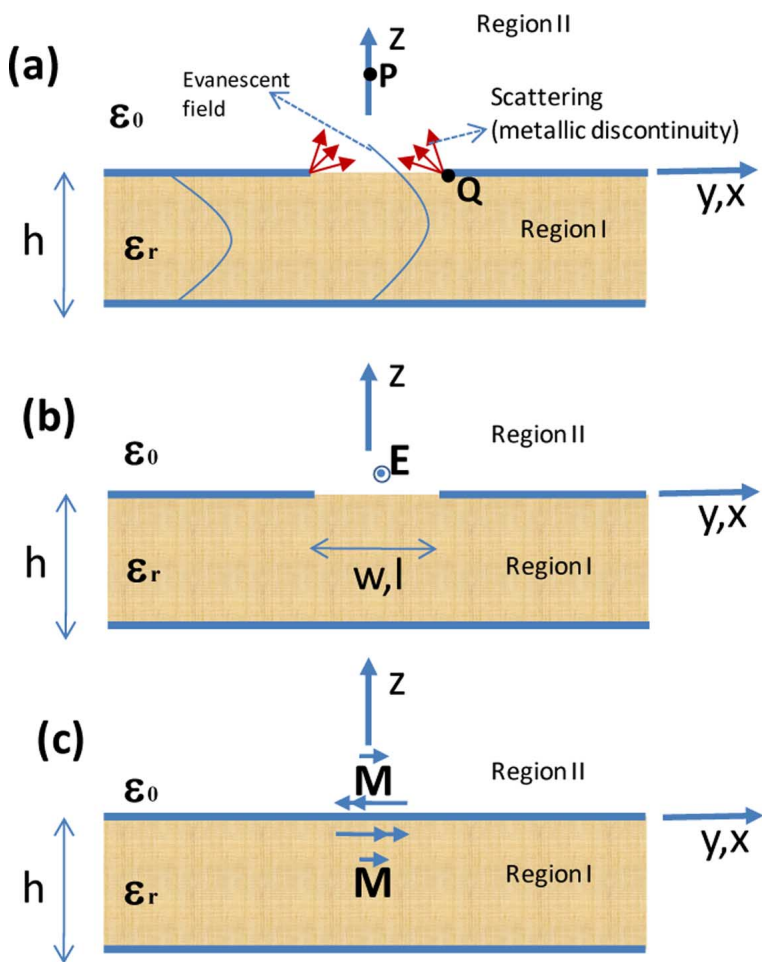

Fig. 2. Cross section ( $x-z$ or $y-z$ plane) of the waveguide of Fig. 1. (a) Fundamental mode profile propagating inside the parallel-plate waveguide and radiation (near field) due to the evanescent field and scattering diffraction of metallic discontinuities above the aperture. (b) Original electromagnetic problem and (c) equivalent problem.

\section{Theory: Parallel-Plate Waveguide With SLOT APERTURE}

Fig. 1 shows the basic structure of an optical slot antennas in a parallel-plate configuration. The substrate (glass) of thickness $h$ has relative permittivity $\varepsilon_{r}$. In order to analyze the effect of parallel-plate waveguide on the optical radiation, we consider the cross section reported in Fig. 2(a): a guided mode propagates along the longitudinal direction, when it arrives at the aperture position the near field will be defined principally by the evanescent field and by the diffraction due to the scattering of the metallic discontinuity. Because of the equivalence principle, the geometry in Fig. 2(b) can be separated into two isolated problems [11] [see Fig. 2(c)] by introducing effective magnetic currents $\vec{M}^{\text {regionII }}=-\vec{M}^{\text {regionI }}$ in the slot areas. In the region above (region I) and in the region below (region II) the top ground plane, the $y, x$-directed magnetic field at $z=0$ excited by a parallel magnetic current $M_{y, x}$ are expressed in terms of the Green's function $G_{y, x}^{\mathrm{I}, \mathrm{II}}$ :

$$
\begin{aligned}
& H_{y, x}^{(\mathrm{I}, \mathrm{II})} \\
& \quad=\iint_{A} G_{y ; x}^{(\mathrm{I}, \mathrm{II})}\left(x-x^{\prime}\right)\left(y-y^{\prime}\right) M_{y, x}^{(\mathrm{I}, \mathrm{II})}\left(x^{\prime}, y^{\prime}\right) d x^{\prime} d y^{\prime}
\end{aligned}
$$

where $A$ indicates the surface of the aperture.

The power specified in both the regions I and II will be defined as

$$
P^{(\mathrm{I}, \mathrm{II})}=-\operatorname{Re}\left\{\iint_{A} H_{y, x}^{*(\mathrm{I}, \mathrm{II})} M_{y, x}^{(\mathrm{I}, \mathrm{II})}(x, y) d A\right\} .
$$

The transverse propagation constant of the modes propagating between the parallel plates are the following

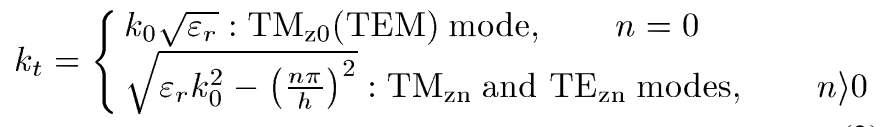

where the wave number $k_{0}$ is given by

$$
k_{0}=\frac{2 \pi}{\lambda_{0}}=\omega \sqrt{\mu_{0} \varepsilon_{0}} .
$$

Here, $\lambda_{0}$ denotes the working wavelength, $\varepsilon_{0}$ and $\mu_{0}$ are the free permittivity and permeability, respectively, and $\omega$ is the angular frequency. Moreover, the equivalent magnetic current generated by the slot is defined as [11]

$$
M_{y, x}^{(\mathrm{I}, \mathrm{II})}\left(k_{x}, k_{y}\right)=\frac{\sin \left(\left(k_{x} w\right) / 2\right)}{\left(k_{x} w\right) / 2} \frac{\cos ^{2}\left(\left(k_{y} l\right) / 4\right)}{(\pi / 2)^{2}-\left(\left(k_{y} l\right) / 4\right)^{2}}
$$

where $k_{x}$ and $k_{y}$ are the wave number along the $x$ - and $y$-direction, respectively. In practice, the fundamental $\mathrm{TM}_{\mathrm{z} 0}$ (TEM) mode is often the only mode of interest. For this purpose, we focus in this paper on the analysis of the slot coupling by means of a $\mathrm{TM}_{\mathrm{z} 0}$ mode: the $\mathrm{TM}_{\mathrm{z} 0}$ (plane wave assumption) can be easily generated by an external source which forces the power in the parallel-plate waveguide [12]; in this manner, the major contribution of the transferred guided power can be assigned to the fundamental mode. As example of evaluation of equivalent magnetic current, we consider glass material $\left(\varepsilon_{r}=5.5\right)$ between the metallic parallel plates.

\section{GME/STRD MODELING}

The radiated electric field at an external point $\mathrm{P}$ of region II of Fig. 2 are expressed as [10]

$$
\begin{aligned}
\mathbf{E}(P)= & \frac{1}{j \omega 4 \pi \varepsilon_{c}} \nabla_{P} \times \nabla_{P} \\
& \times\left(\int_{V} \mathbf{J}_{i} G\left(\mathbf{r}, \mathbf{r}^{\prime}\right) d V_{Q}\right. \\
& \left.+\int_{S}(\mathbf{H} \times \hat{n}) G\left(\mathbf{r}, \mathbf{r}^{\prime}\right) d S_{Q}\right) \\
& -\frac{1}{4 \pi} \nabla_{P} \times\left(\int_{S}(\hat{n} \times \mathbf{E}) G\left(\mathbf{r}, \mathbf{r}^{\prime}\right) d S_{Q}\right)-\frac{\mathbf{J}_{i}}{j \omega \varepsilon_{c}}
\end{aligned}
$$




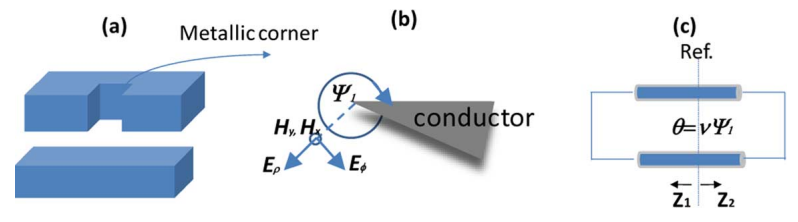

Fig. 3. (a) Metallic corner of a slot aperture in parallel-plate waveguide. (b) Electromagnetic field configuration for a propagating $\mathrm{TM}_{\mathrm{zn}}$ mode along the $y$ and $x$-direction. (c) STRD transmission line modeling of the metallic corner.

where in spherical coordinates, the slot coordinate is at $\mathrm{Q}=$ $\mathrm{Q}\left(\mathrm{r}^{\prime}, \theta^{\prime}, \varphi^{\prime}\right)$, and the field is evaluated at $\mathrm{P}=\mathrm{P}(\mathrm{r}, \theta, \varphi) ; \varepsilon_{\mathrm{c}}$ indicates the cladding permittivity (permittivity of region II), and $\mathbf{M}=\mathbf{n} \times \mathbf{E}$ represents the equivalent magnetic current distribution of (5). In spherical coordinates, the Green function satisfies the Helmholtz scalar equation and the multipole expansion of the Green's function of order $s$ given by [13]-[20]

$$
G\left(\mathbf{r}, \mathbf{r}^{\prime}\right)=\sum_{s, m} g_{s}\left(r, r^{\prime}\right) Y_{s, m}^{*}\left(\theta^{\prime}, \varphi^{\prime}\right) Y_{s, m}(\theta, \varphi)
$$

We observe that (7) presents a discontinuity peak of the first derivative at the point $\mathrm{Q}$ [point of singularity related to the metallic discontinuity of Fig. 2(a)]. According to this property, the MEG is suited for near-field evaluation near metallic singularities. Moreover, index $s$ represents the order of the expansion and represents the accuracy of the solution: the convergence of the near-field solution can be performed by increasing the order of MEG.

Concerning the convergence of the solution of the near field, an accurate study of the metallic discontinuity of the slot region is necessary. As illustrated in Fig. 3(a), each side of the slot aperture is characterized by metallic corners. According to Fig. 2(b), the slot problem requires an electric field oriented along the $x$ and $y$-direction: along both the directions the $\mathrm{TM}_{\mathrm{zn}}$ modes are defined by the following components [21]:

$$
\begin{aligned}
E_{\rho} & =-j \omega \mu \frac{1}{\rho} \frac{\partial \Phi}{\partial \phi} \\
E_{\phi} & =j \omega \mu \frac{\partial \Phi}{\partial \rho} \\
H_{x, y} & =k^{2} \Phi
\end{aligned}
$$

where $\Phi$ is a suitable Hertzian electric potential function [10]. The metallic corner of the top ground plane defines the angle $\Psi_{1}$ [see Fig. 3(b)] which is related to the shape geometry of the metallic discontinuity. The configuration of Fig. 3(b) allows to define, by means of the STRD model [10], the behavior of the electromagnetic fields around the discontinuity (important aspect of the near-field evaluation). The angle $\Psi_{1}$ defines the transmission line configuration of Fig. 3(c). By applying the resonance condition $\mathrm{Z}_{1}+\mathrm{Z}_{2}=0$ (where $\mathrm{Z}_{1}$ and $\mathrm{Z}_{2}$ are the impedances calculated starting from a reference plane) at the reference plane of Fig. 3(c), we calculate the electromagnetic field singularity $\nu[10]$.

We summarize in Table I the electromagnetic field behavior and the singularities $\nu$ for different type of metallic wedges. We observe that the case of thin conducting layer $\left(\Psi_{1}=2 \pi\right)$

\begin{tabular}{|c|c|c|c|}
\hline Geometry & $v$ & $E_{\rho}, E_{\phi}$ & $H_{y}, H_{x}$ \\
\hline 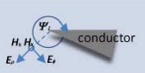 & $v=\pi / \Psi_{i}$ & $\propto \rho^{v-1}$ & $\propto \rho^{v}$ \\
\hline 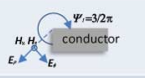 & $v=2 / 3$ & $\propto \rho^{-1 / 3}$ & $\propto \rho^{2 / 3}$ \\
\hline 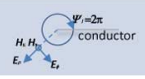 & $v=1 / 2$ & $\propto \rho^{-1 / 2}$ & $\propto \rho^{1 / 2}$ \\
\hline
\end{tabular}
correspond to the case of surface plasmon waves.
TABLE I

$\mathrm{TM}_{\mathrm{zn}}$ Field Behavior Around the Metallic Corners

\section{GME NEAR-FIELD AND FAR-FIELD EVALUATION: RESUlts AND DISCUSSIONS}

By implementing (FORTRAN code implementation) the electromagnetic field of Table I, the equivalent magnetic current of (5), and the MEG (7) in (6), it is possible to evaluate the near field generated by the slot of Fig. 1. The total near field $|\mathrm{E}|$ versus the $z$-direction is obtained by integrating (7) over and $\phi$ and by supposing the impressed currents $\mathbf{J}_{\mathrm{i}}=0$. Moreover, as in many practical cases, we consider in our analysis metallic discontinuities with $\Psi_{1}=3 / 2 \pi$ and plane wave as source. In order to analyze the convergence of the solution with respect to the order of the MEG, we fix the parameters $w=10 \mu \mathrm{m}$, $l=30 \mu \mathrm{m}$, and $\lambda_{0}=1.55 \mu \mathrm{m}$. Fig. 4(a)-(c) shows that a good convergent near-field solution can be performed by considering MEG orders of $s=6$ and $s=8$. The low-order $s=1$ does not provide the exact trend of the near field in proximity of the slot aperture and may give wrong near-field results. This aspect is very important for near-field detection systems.

By changing the slot dimension, it is possible to tune the maximum distance of the near-field detection. In particular, we consider in the example of Fig. 5 three slot configurations: the near-field region changes of about $1 \mu \mathrm{m}$ by considering geometrical variations of the order of $10 \mu \mathrm{m}$. We observe that the electric field contributions in an external point $\mathrm{P}$ defined along the $z$ axis are generated by the radiation of the four wedges, consecutively, for small apertures, the total electric field exhibits numerical noise (due to singularity region). This numerical noise can be decreased by increasing the order $s$ (see in Fig. 4 the curve with $s=8$ ).

Regarding the far-field evaluation, we calculate (6) and (7) in far field region. In order to validate the MEG method, we compare the results with a properly designed 3-D FEM tool typically used for the design of micrometric structures [22]. The FEM near-field region is the region closest to the source (parallel-plate waveguide with aperture), and the electric field $\mathbf{E}(x, y, z)$ that is external to the region bounded by a closed spherical surface may be written as [23]

$$
\begin{aligned}
\mathbf{E}(x, y, z)=\int_{S}\left(\left(j \omega \mu_{0} \mathbf{H}_{\text {tan }}\right) G\right. \\
\left.\quad+\left(\mathbf{E}_{\text {tan }} \times \nabla G\right)+\left(\mathbf{E}_{\text {normal }} \nabla G\right)\right) d S
\end{aligned}
$$

where $S$ represents the radiation boundary surface, $j$ is the imaginary unit, $\mathbf{H}_{\text {tan }}$ is the component of the magnetic field that is tangential to the surface, $\mathbf{E}_{\text {normal }}$ is the component of 

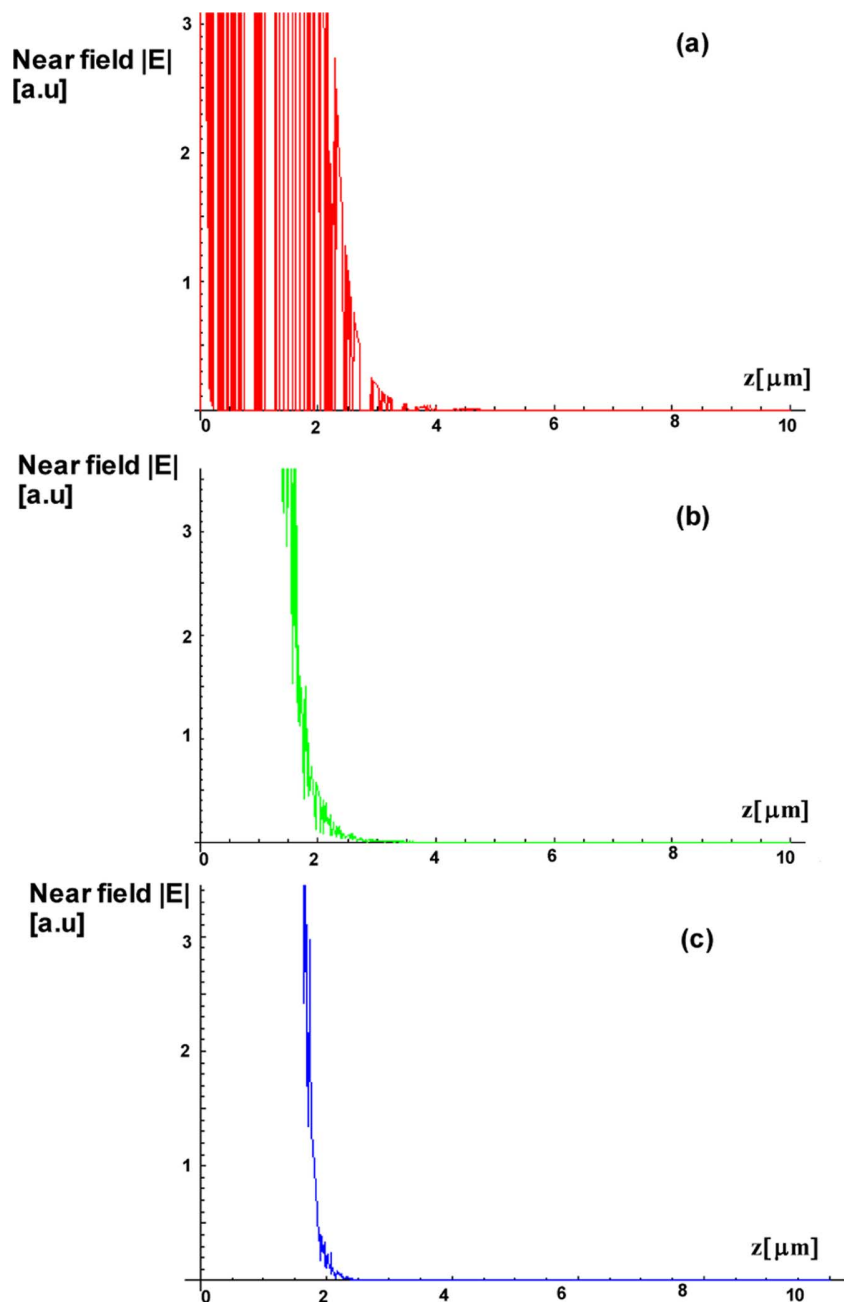

Fig. 4. Near-field evaluation versus $z$ for (a) $s=1$, (b) $s=6$, and (c) $s=8$.

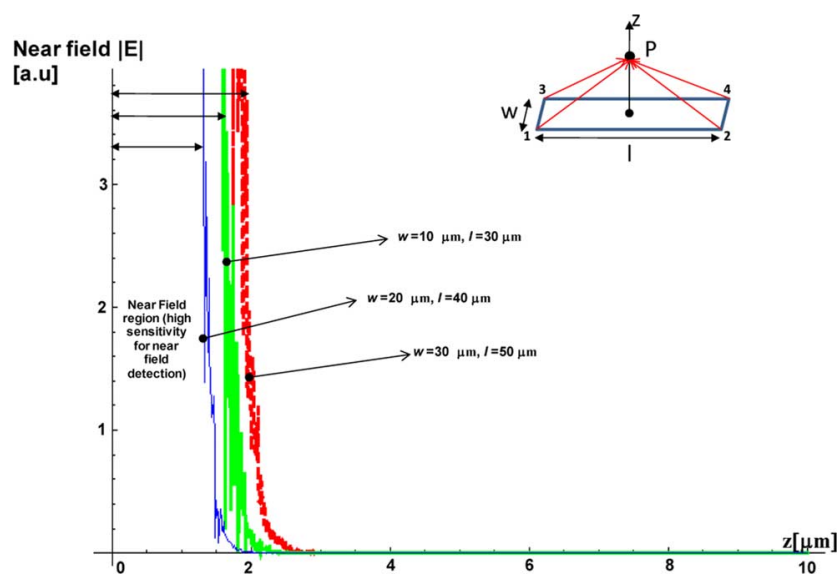

Fig. 5. Near-field evaluation for different slot sizes. The working wavelength is $\lambda_{0}=1.55 \mu \mathrm{m}$, and $s=6$. Inset: electric field contributions in an external point $P$. The contributions are due to the four wedges of the rectangular aperture.

the electric field that is normal to the surface, and $G$ is the free space Green's function, given by

$$
G=\frac{e^{-j k_{0}\left|\mathbf{r}-\mathbf{r}^{\prime}\right|}}{\left|\mathbf{r}-\mathbf{r}^{\prime}\right|}
$$

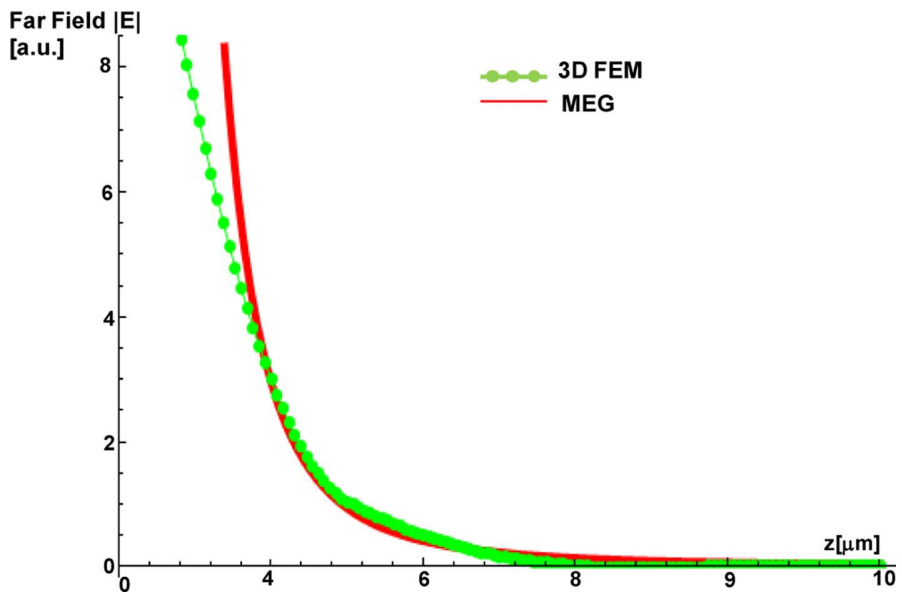

Fig. 6. Comparison between 3-D FEM and MEG far field at $\lambda_{0}=1.55 \mu \mathrm{m}$ for a slot aperture with $w=10 \mu \mathrm{m}, l=30 \mu \mathrm{m}$.

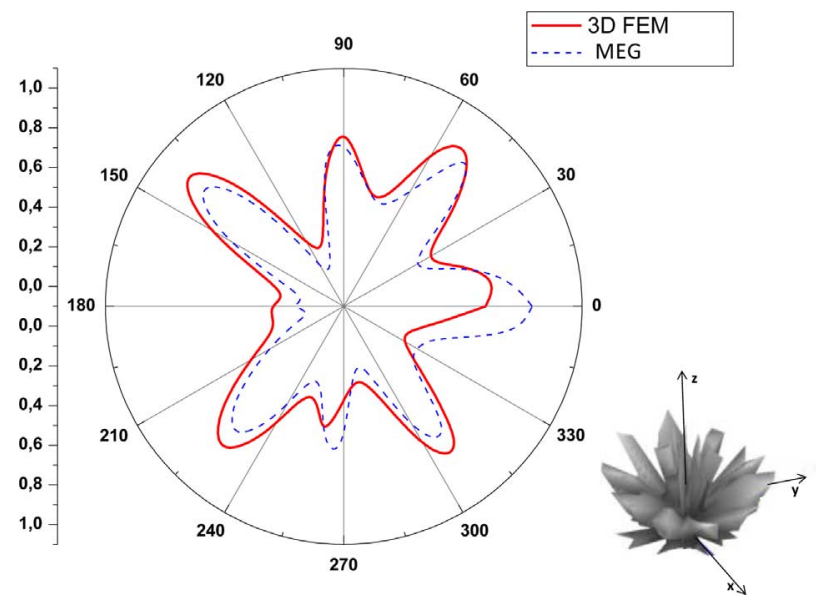

Fig. 7. Comparison between MEG and 3-D FEM methods: far-field radiation pattern versus $\phi$ for $\theta=10^{\circ}$. Inset: 3-D FEM radiation pattern. The simulated slot aperture is characterized by $w=10 \mu \mathrm{m}, l=30 \mu \mathrm{m}$, and $\lambda_{0}=1.55 \mu \mathrm{m}$.

being $k_{0}$ the free space wave number, and $\mathbf{r}$ and $\mathbf{r}^{\prime}$ representing field points and source points on the surface, respectively. Figs. 6 and 7 show good agreement between 3-D FEM and MEG approach concerning the total far field $|\mathrm{E}|$ versus $z$, and the far-field radiation pattern calculated along the lobe of maximum radiation, respectively.

We note from Fig. 6 that for distance of $z>4 \mu \mathrm{m}$, the farfield radiation pattern of Fig. 7 becomes stable (convergence of the far-field solution); for this reason, we fix as observation point of the far-field radiation pattern of Fig. 7 a distance $z=$ $10 \mu \mathrm{m}$. The far-field radiation pattern of Fig. 7 is equal also for distances $z>10 \mu \mathrm{m}$. Moreover, we observe that the MEG method requires a low computational cost if compared with the 3-D FEM one: the MEG provides a good convergent solution with a low (CPU)-time, by decreasing the computational time of 13 times as reported in Table II $(2.3$ and $30 \mathrm{~h}$ are requested for the MEG and the 3-D FEM simulation, respectively, by using a 1-GHz PC, 512/M-RAM). The accuracy of the solution and the low computational cost prove that the proposed MEG method is suitable for electromagnetic simulators. 
TABLE II

COMPUTATIONAL TIME FOR THE EXAMPLE OF Fig. 7

\begin{tabular}{|l|l|}
\hline 3 D FEM & MEG \\
\hline$\cong 30 \mathrm{~h}$. & $\cong 2.3 \mathrm{~h}$. \\
\hline
\end{tabular}

(a)

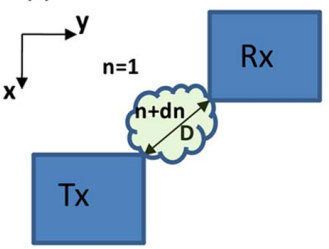

(c)

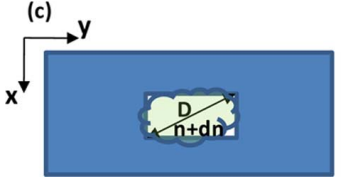

(b)

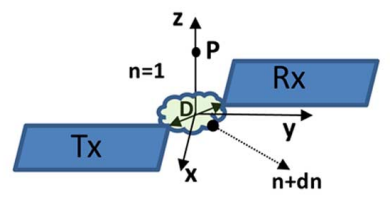

(d)

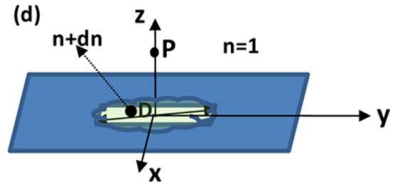

Fig. 8. Layouts related to the detection of dielectric contrasts $d n$ : (a) $90^{\circ}$ metallic corners in the $(x, y)$ plane and (b) in the 3-D space; (c) rectangular metallic aperture in the $(x, y)$ plane and (d) in the 3-D space.

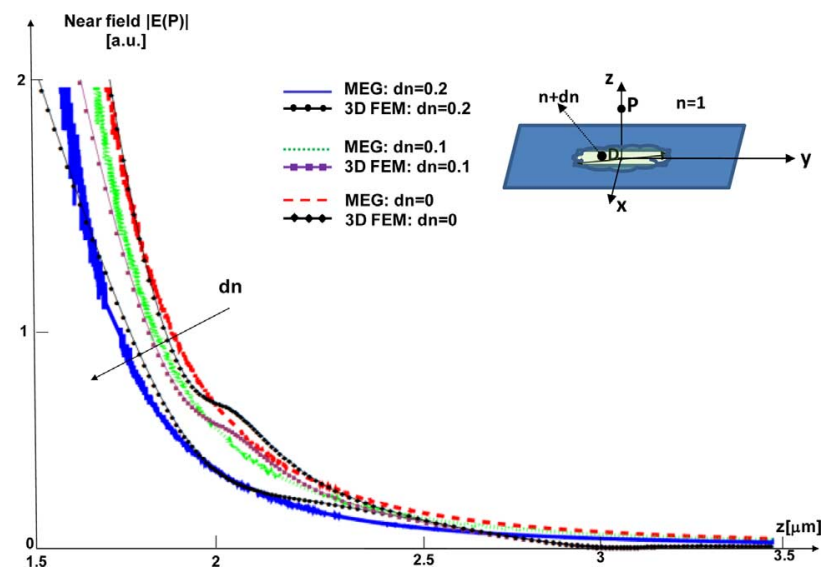

Fig. 9. Dielectric contrast detection: comparison between MEG and 3-D FEM total electric near field versus a point $\mathrm{P}$ defined along the vertical $z$ axis and for different variations of refractive index. The MEG order of the MEG expansion is $s=8$.

\section{NEAR-FiELD APPliCATION: LIGHT ENHANCEMENT FOR PERMitTIVITY SENSING}

Metallic wedges are suitable for dielectric permittivity sensing because they can focus the energy in proximity of the discontinuity and they behave as plasmon probes for optical detection systems [24]. An example of optical field detection system is reported in Fig. 8(a) and (b) where a transmitter metallic wedge $\mathrm{T}_{\mathrm{x}}$, excited by a surface optical wave, radiates the focused electromagnetic field, and a receiver wedge probe $R_{\mathrm{x}}$ detects the variation of the refractive index (dielectric contrast $d n$ ) in a background with refractive index $n$ [24]. The distance and the orientation of both probes are defined in order to distinguish without errors the refractive index variation. In order to enhance the radiated field in an external point $\mathrm{P}$, useful to detect a variation $d n$, we consider the effect of four wedges of the rectangular aperture illustrated in Fig. 8(c) and (d).

Fig. 9 proves that the MEG method allows to distinguish the near-field amplitudes also for low dielectric contrasts. Moreover, Fig. 9 validates the convergence of the MEG approach by

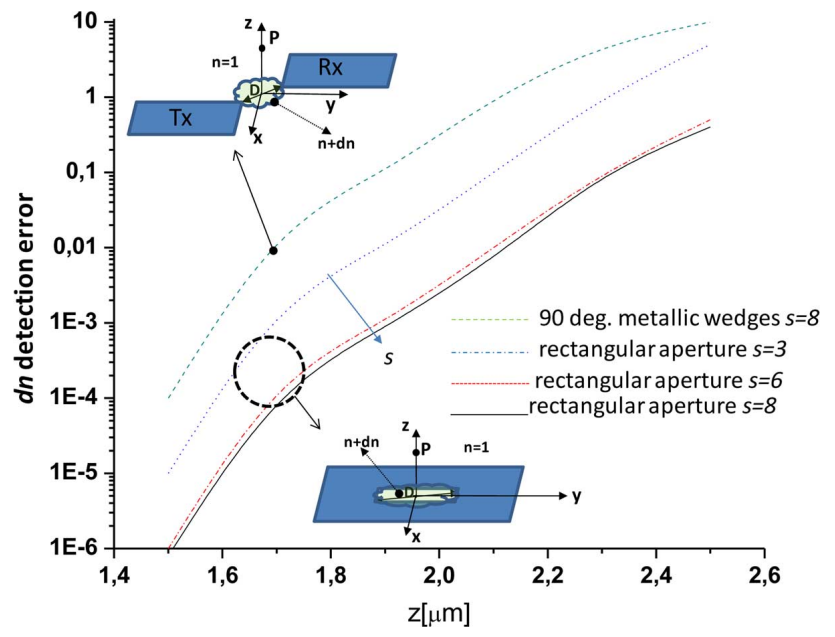

Fig. 10. Comparison of $d n$ detection error versus $z$ for different values of the MEG order $s$. The distance $D$ (diagonal of the rectangular aperture) is about $31.6 \mu \mathrm{m}$.

means of the comparison with 3-D FEM result: good agreement between 3-D FEM and MEG results is found. As illustrated in Fig. 10, the $d n$ sensitivity numerically increases by increasing the order $s$. Consecutively, the numerical error will decrease by increasing the MEG order $s$.

During the simulations, we have observed that the detection error increases with the order $s$ and arrives to a limit case corresponding to a minimum error at $s=8$ (the plots of Fig. 10 with $s=9,10,11 \ldots$ will coincide with the plot calculated at $s=8)$. Moreover, Fig. 10 shows the comparison between the sensitivity errors of the layouts reported in Fig. 8(a) and (c): by fixing the order $s=8$, the rectangular aperture layout exhibits a numerical error of two order lower if compared with the numerical error of the $90^{\circ}$ metallic wedge layout.

Being the limit case of the $90^{\circ}$ metallic wedge system (corresponding to a minimum error of $d n$ detection) also convergent at $s=8$, this result highlights the advantage of the rectangular aperture for an high sensitivity permittivity detection system based on the near-field enhancement. This advantage is also physically explained by the four near-field contributions corresponding to the four metallic corners which provide a stronger electric field if compared with the case of two metallic corner contributions related to the $90^{\circ}$ metallic wedge system.

\section{FAR FIELD: COMPARISON WITH EXPERIMENTAL RESULTS}

In order to validate the proposed MEG method, we measure the radiation field of a rectangular aperture of a silver parallel-plate waveguide which enclose glass material $\left(\varepsilon_{r}=5.5\right)$. The used experimental setup is reported in the inset of Fig. 11. A light probe beam (from a tungsten broad band lamp) is launched from a tapered fiber and directly injected into the parallel-plate waveguide. In order to improve a plane wave source, the tapered fiber is not attached to the waveguide. The light exiting the waveguide and radiated by the rectangular slot (the slot aperture is characterized by $w=50 \mu \mathrm{m}, l=300 \mu \mathrm{m}$, and is obtained by means of a controlled needle system) is collected and collimated by a multimode fiber connected to an N-cooled InGaAs optical multichannel analyzer (OMA). The multimode fiber behaves as a receiving probe positioned directly above the aper- 


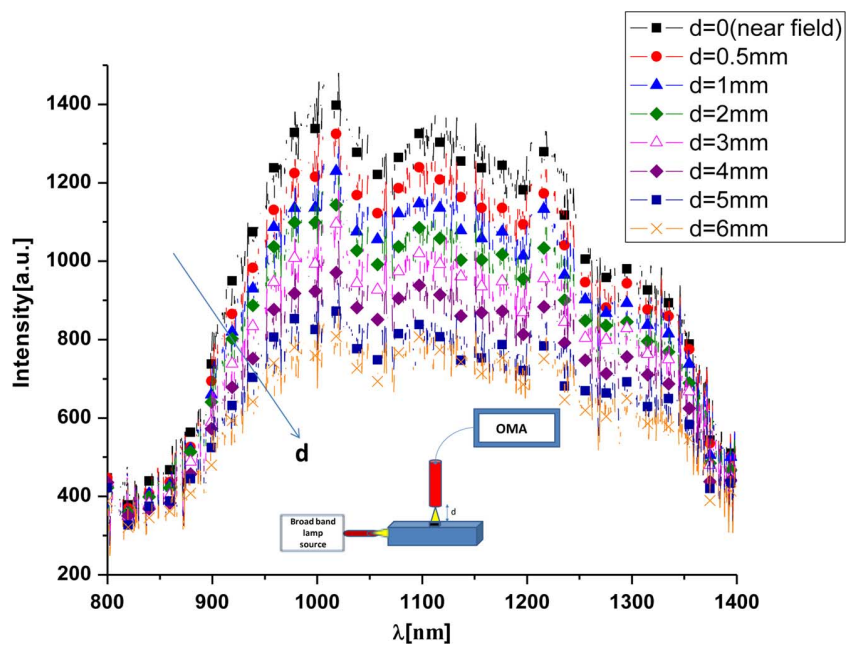

Fig. 11. Measured spectra of radiation field obtained by increasing the distance $d$. The efficiency decreases by increasing the distance $d$. Inset: experimental setup for optical radiated far field.

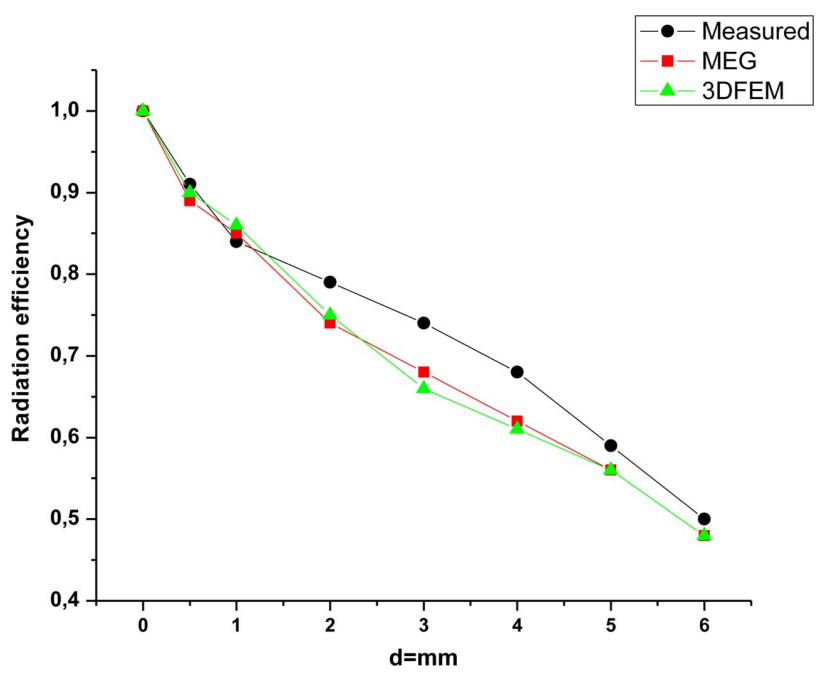

Fig. 12. Comparison of radiation efficiency between measured MEG and 3-D FEM results versus $d$. The measurement and the simulations are performed at the working wavelength $\lambda_{0}=1 \mu \mathrm{m}$.

ture. In Fig. 11, the spectra of the radiated field for different positions $\mathrm{d}$ of the receiver probe are illustrated. The position $d=0$ corresponds to the case of probe fiber attached to the top ground plate. In order to estimate the radiation efficiency, we consider as intensity reference the intensity measured at $d=0$ as (we normalize to the unit the maximum intensity measured at $\left.\lambda_{0}=1 \mu \mathrm{m}\right)$, and we calculate the ratio between the intensities $[\operatorname{Intensity}(d) / \operatorname{Intensity}(d=0)]$. Fig. 12 shows good agreement between 3-D FEM, MEG, and experimental radiation efficiency by confirming the accuracy of the proposed approach. We observe that the slot size and the working wavelength are chosen according to the accuracy of the needle system and to OMA spectrum, respectively.

\section{CONCLUSION}

We present in this work an accurate modeling based on multipole expansion of the Green's function which allows to calcu- late the near and far field generated by a rectangular micrometric aperture on parallel-plate waveguide.

We validate the importance of the rectangular aperture layout by considering as application a near-field permittivity detection system and comparing the detection sensitivity of different layouts.

Regarding the far-field characterization, good agreement between 3-D FEM and experimental results is observed. The proposed approach is suited for the design of near-field/far-field wireless detection systems and for nanoantenna applications including biosensors obtained by functionalizing the surface corresponding the rectangular aperture.

\section{REFERENCES}

[1] D. J. Michalak, S. R. Amy, D. Aureau, M. Dai1, A. Estève, and Y. J. Chabal, "Nanopatterning Si(111) surfaces as a selective surface-chemistry route," Nature Nanotech., vol. 9, pp. 266-271, Jan. 2010.

[2] H. Jin, D. A. Heller, M. Kalbacova, J.-H. Kim, J. Zhang, A. A. Boghossian, N. Maheshri, and M. S. Strano, "Detection of single-molecule $\mathrm{H}_{2} \mathrm{O}_{2}$ signalling from epidermal growth factor receptor using fluorescent single-walled carbon nanotubes," Nature Nanotech., vol. 5, pp. $1-8,2010$.

[3] M. A. C. Stuart, W. T. S. Huck, J. Genzer, M. Müller, C. Ober, M. Stamm, G. B. Sukhorukov, I. Szleifer, V. V. Tsukruk, M. Urban, F. Winnik, S. Zauscher, I. luzinov, and S. Minko, "Emerging applications of stimuli-responsive polymer materials," Nature Nanotech., vol. 9, pp. 101-113, 2010.

[4] D. R. Jackson, J. Chen, R. Qiang, F. Capolino, and A. A. Oliner, "The role of leaky plasmon waves in the directive beaming of light through a subwavelength aperture," Opt. Exp., vol. 16, no. 26, pp. 21271-21281, 2008.

[5] M. F. Garzia-Parajo, "Optical antennas, focus on biology," Nature Photon., vol. 2, pp. 201-203, 2008.

[6] H. Fischer and O. J. F. Martin, "Engineering the optical response of plasmonic nanoantennas," Opt. Exp., vol. 16, no. 12, pp. 9144-9154, 2008.

[7] A. O. Boryssenko, J. Liao, J. Zeng, S. Deng, V. M. Joyner, and Z. R. Huang, "Radio-optical dual-mode communication modules integrated with planar antennas," IEEE Trans. Microw. Theory Tech., vol. 58, no. 2, pp. 403-410, Feb. 2010.

[8] E. R. L. Lara, W. Fu, K. Ghorbani, W. S. T. Rowe, and A. Mitchell, "Reconfigurable two-arm spiral antenna microwave photonic polarization diversity technique," IEEE Photon. Technol. Lett., vol. 21, no. 22, pp. 1668-1670, Nov. 2009.

[9] E. Hamidi and A. M. Weiner, "Post-compensation of ultra-wideband antenna dispersion using microwave photonic phase filters and its applications to UWB systems," IEEE Trans. Microw. Theory Tech., vol. 57, no. 4, pp. 890-898, Apr. 2009.

[10] A. Massaro, L. Pierantoni, R. Cingolani, and T. Rozzi, "A new analytical model of diffraction by 3D-dielectric corners," IEEE Trans. Antennas Propag., vol. 57, no. 8, pp. 2323-2330, Aug. 2009.

[11] R. Marg, A. Mallwitz, and A. F. Jacob, "Radiation efficiency of apertures in parallel-plate waveguides," Microw. Opt. Technol. Lett., vol. 21, no. 3, pp. 159-162, 1999.

[12] R. Shavit, "Impedance characteristics of a slot antenna fed by a parallel-plate waveguide," Microw. Opt. Technol. Lett., vol. 14, no. 2, pp. 126-128, 1997.

[13] V. I. Okhmatovski and A. C. Cangellaris, "Efficient calculation of the electromagnetic Dyadic Green's function in spherical layered media," IEEE Trans. Antennas Propag., vol. 51, no. 12, pp. 3209-3220, Dec. 2003.

[14] C. G. Someda, Onde Elettromagnetiche. Torino, Italy: UTET, 1996.

[15] Y. Chen, T. L. Simpson, and T. Q. Ho, "Highly efficient technique for solving radiation and scattering problems," Proc. Inst. Elect. Eng. H, vol. 139 , no. 1 , pp. $7-10,1992$.

[16] J. S. Gardner, "Uniform asymptotic expansion of the associated Legendre function to leading term for complex degree and integral order," IEEE Trans. Antennas Propag., vol. 55, no. 6, pp. 1797-1803, Jun. 2007. 
[17] M. S. Narasimhan and S. Christopher, "On expansion of cylindrical Bessel function," IEEE Trans. Antennas Propag., vol. AP-32, no. 11, pp. 1268-1270, May 1984.

[18] R. Cicchetti and A. Faraone, "Analysis of open-ended circular waveguide using physical optics and incomplete Hankel functions formulation," IEEE Trans. Antennas Propag., vol. 55, no. 6, pp. 1887-1892, Jun. 2007.

[19] P. M. Morse and H. Feshbach, Methods of Theoretical Physics, 5th ed. New York: McGraw-Hill, 1953, pp. 1273-1283.

[20] G. R. Hadley, "High-accuracy finite_difference equations for dielectric waveguide analysis II: Dielectric corner," J. Lightw. Technol., vol. 20, no. 7, pp. 1221-1231, Jul. 2002.

[21] T. Rozzi and M. Mongiardo, Open Electromagnetic Waveguides. London, U.K.: IEE, 1997.

[22] A. Massaro, V. Errico, T. Stomeo, A. Salhi, R. Cingolani, A. Passaseo, and M. De Vittorio, "3D FEM modeling and fabrication of circular photonic crystal microcavity,” J. Lightw. Technol., vol. 26, no. 16, pp. 2960-2968, Aug. 2008.

[23] S. Caorsi, D. Moreno, and F. Sidoti, "Theoretical and numerical treatment of surface integrals involving the free-space Green's function," IEEE Trans. Antennas Propag., vol. 41, no. 9, pp. 1296-1301, Sep. 1993.

[24] A. Massaro and R. Cingolani, "STRD and near field modeling of metallic wedges for optical detection systems," J. Lightw. Technol., vol. 28 , no. 24 , pp. $3562-3568$, Dec. 2010 .
Alessandro Massaro received the Laurea degree in electronic engineering and the Ph.D. degree in telecommunication engineering from the Università Politecnica delle Marche, Ancona, Italy, in 2001 and 2004, respectively.

From 2004 to 2006, he was a Research Scientist (Postdoc) in the Department of Electromagnetism and Bioengineering, Università Politecnica delle Marche. In 2006, he spent two years in Research and Development at medical and industrial optics industry (endoscope design and optical systems). He was the Principal Investigator at the National Nanotechnology Laboratory of CNR-INFM for two years. He is currently the Team Leader in Robotics Lab. 1 of the Center of Biomolecular Nanotechnology, Italian Institute of Technology, Arnesano, Lecce, Italy. His research interests include the design and modeling of photonic bandgap circuits, the development of computer-aided design tools in the area of integrated optics, microelectromechanical systems/nanoelectromechanical systems technology and systems, and smart material modeling.

Dr. Massaro is a member of the European Microwave Association.

Roberto Cingolani was born in Milan, Italy, in December 1961. He received the Ph.D. degree in physics from Scuola Normale Superiore di Pisa, Pisa, Italy.

From 1989 to 1991, he was a Staff Member of Max Planck Institute FKFStuttgart. He was a Visiting Scientist at Tokyo University in 1997, and a Full Professor of experimental physics at University of Salento in 2000. Since 2004, he has been the Scientific Director of the Italian Institute of Technology, Genova, Italy. He is the author or coauthor of more than 500 papers in international journals and holds 20 patents. His research interests include nanotechnology and nanofabrication, semiconductor physics and devices, molecular organic nanostructures and devices, and bionanotechnologies. 